\title{
Mohamad Hayssam ElFawal MD, FACS, IFASMBS
}

\section{Mohamad Hayssam ElFawal ${ }^{1}$}

Published online: 9 December 2019

(C) Springer Science+Business Media, LLC, part of Springer Nature 2019

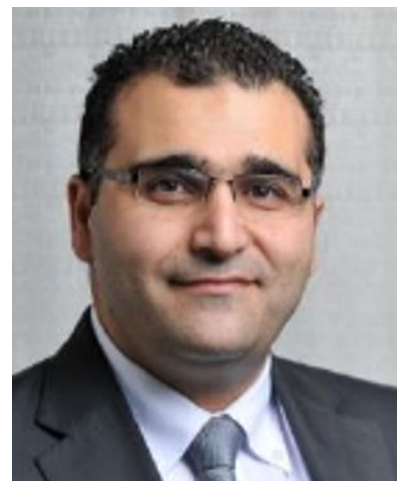

Dr. Mohamad Hayssam ElFawal MD, FACS, IFASMBS, is a master surgeon in minimally invasive metabolic and bariatric surgery. He received his general surgery diploma from the American University of Beirut, Lebanon. Dr. ElFawal completed a fellowship in Hepato-Biliary and Transplant Surgery at Lille University in France.

Dr. ElFawal has extensive experience in the treatment of obesity and metabolic syndrome, and he currently serves as the president of the Pan Arab Society of Bariatric and Metabolic Surgery. $\mathrm{He}$ is also the program director of the International Bariatric Club Mini Fellowship. Dr. ElFawal is a clinical assistant professor of surgery at Beirut Arab University. He is active in the International Federation for the Surgery of Obesity and Metabolic Disorders Middle East North Africa Chapter (IFSOMENAC) where he served on the corporate and finance committee and is an executive board member and treasurer.

Dr. ElFawal was the chairman of the Bariatric and Metabolic Unit at Makassed General Hospital in Beirut, Lebanon, from 2014 to 2018. Since 2013, Dr. ElFawal is the CEO of the Bariatric Surgery Clinic, a center that applies a multidisciplinary approach for bariatric surgery. He is known to be dedicated to

Mohamad Hayssam ElFawal

hayssamfawal@gmail.com

1 Pan Arab Society of Bariatric and Metabolic Surgery, Beirut, Lebanon caring for his patients through a holistic approach to health and well-being.

Dr. ElFawal has been a fellow of the American College of Surgeons since 2011 and an international fellow of the American Society of Metabolic and Bariatric Surgery since 2015. He became a master surgeon in metabolic and bariatric surgery in 2017.

In 2014, Dr. Hayssam ElFawal and his team performed a gastric sleeve surgery using the paravertebral block anesthesia (PBA) technique with live transmission from Makassed General Hospital to the IFSO 2014 Congress in Montreal, Canada. This was followed by a live transmission of the same procedure to the IFSO 2017 Congress held in London. That same year, Dr. ElFawal performed the first one anastomosis gastric bypass in the world under PVB.

Dr. ElFawal has published many articles on hepatobiliary and bariatric surgery. He has more than 50 international presentations and has been a guest speaker in more than 80 national and international meetings.

Despite the fact that Dr. ElFawal lived and worked in a small country (Lebanon) that passed through 20 years of civil war, he has been able to set an example of excellence in surgery by providing the multidisciplinary team (MDT) in bariatric surgery since 2010 and has trained more than 100 surgeons from Middle East and North Africa (MENA) region on different bariatric procedures with MDT approach.

He was inspired by his mentor, Professor Ali Raad, exgovernor of the American College of Surgeons in Lebanon and one of the first American board members in the MENA region since 1965 .

Dr. ElFawal has been married for 20 years to Dr. Bouchra Berro one of the pioneers of pediatric endocrinology in Lebanon. They have raised four children, including Karim who is 19 years old, and a 2nd year pre-medical student at the American University of Beirut. Karim's ambition is to have his biography also published in obesity surgery in the next 30 years. Dr. ElFawal is also the father of Rim, Lynn, and Sara - all college students with medical and architect preferences. Dr. ElFawal adores spending his time with family, playing table tennis, and watching soccer and basketball games. 